\title{
Dissociating object- and response-based components of negative priming through effects of practice
}

\author{
GEORGE HOUGHTON and PALOMA MARÍ-BEFFA \\ University of Wales, Bangor, Wales
}

\begin{abstract}
The negative priming (NP) effect is the slowing of responses to an imperative stimulus (probe) that has recently been ignored (prime). Prevailing accounts of the phenomenon attribute it to a variety of causes, all centered on a representation of the stimulus event itself. However, we argue that the most commonly used NP paradigms confound stimulus- and response-based factors. In two experiments, we demonstrate the importance of response factors in producing NP and show clear empirical dissociations between object- and response-centered NP when studying their time courses over extended practice. When distractors compete for a response (response-based), the NP effect is both more robust and more resistant to the effects of practice. On the other hand, when prime distractors do not compete for response (object-based), they yield weaker NP effects that disappear with practice. We conclude that the NP effects shown in the most common procedures are produced by a combination of distinct factors that tend to act in the same direction.
\end{abstract}

Practiced actions can be evoked and executed in a rather automatic fashion, such as when we turn on a light switch on entering a familiar room, even in daylight. Sometimes, such automatisms have to be avoided to prevent them from interfering with other objectives. For instance, a British driver (used to right-hand-drive cars) renting a left-handdrive car when abroad may find his/her left hand still reaching for the gearshift, only to end up groping the door handle. Thus, as well as defining the desired end goal of an action (get hold of the gearshift), intentional behavior sometimes requires the suppression of automatic responses that are contextually inappropriate.

In experimental psychology, the activation and control of contextually irrelevant representations are usually studied with a variety of selective attention paradigms. Several studies have shown that to-be-ignored stimuli (distractors) automatically activate several kinds of representation, such as physical and semantic ones (Marcel, 1983; Underwood, 1976), as well as their associated responses (De Jong, Liang, \& Lauber, 1994; DeSoto, Fabiani, Geary, \& Gratton, 2001; Eimer \& Schlaghecken, 1998; B. A. Eriksen \& C. W. Eriksen, 1974; Miller, 1991). This implies that, for the control of a goal-directed action, attentional selection can take place at many levels of representation from perception to action (Tipper, Weaver, \& Houghton, 1994).

Two mechanisms are commonly proposed to achieve this attentional control: One boosts the level of activation

We are grateful to Sarah Grison and two anonymous reviewers for their very helpful comments on this article. Correspondence should be addressed to G. Houghton, School of Psychology, University of Wales, Bangor, Gwynedd, LL57 2DG Wales (e-mail: g.houghton@bangor .ac.uk). of task-relevant information with respect to background levels (see, e.g., Desimone \& Duncan, 1995; Houghton \& Tipper, 1994), whereas another can specifically suppress irrelevant, nontarget information (Bowman, Schlaghecken, \& Eimer, 2006; Caputo \& Guerra, 1998; Cepeda, Cave, Bichot, \& Kim, 1998; Gernsbacher \& Faust, 1991; Houghton \& Tipper, 1994, 1996; Orekhova, Stroganova, \& Posikera, 2001; Tipper, 1985; Watson \& Humphreys, 1997; West \& Alain, 2000). One means by which the consequences of inhibitory selection are studied is with the negative priming (NP) procedure (Tipper, 1985). The logic of this procedure is that if the internal representations of a distractor in a prime display are inhibited, then processing of a subsequent object (in a probe or target display), which makes contact with these inhibited representations, will be impaired (e.g., slowed). For example, when participants are required to respond to one of two simultaneously presented letters (e.g., $A, B$ ), then during the selection of a target letter (say, $A$ ), information regarding the distractor letter $(B)$ may be subject to inhibition. If so, subsequent processing of the same letter $(B)$, which is assumed to require access to the inhibited representation, will be impaired.

This result has been observed in many experimental procedures and has given rise to a number of different accounts (Houghton \& Tipper, 1994; Milliken, Joordens, Merikle, \& Seiffert, 1998; Neill, Valdes, Terry, \& Gorfein, 1992; Tipper, 1985; Tipper \& Cranston, 1985). The theories of Houghton and Tipper concentrate on the role of inhibitory mechanisms of selective attention in processing the prime. In Houghton and Tipper's (1994) theory, perceptual object assemblies activated by distractors enter inhibited states when external activation ceases (see Tipper, 2001, for further discussion of the theory). In Neill 
et al.'s episodic theory, (episodic) perceptual representations of distractors are tagged in some manner as nontargets; in Milliken et al.'s temporal integration account, current perceptual contents (i.e., in the probe trial) need to be integrated (or not) with an episodic record of the prime stimulus. The important point here is that each account emphasizes processes centered on a representation of the stimulus event. In this article, we wish to draw attention to the fact that, in the standard NP design (as described above, more examples are given below), the to-be-ignored distractor in the prime is associated with a response-either long term or established during practice for the experiment - that is incompatible with that to the target. In the example given above, the distractor letter $B$ in the prime has a response associated with it, which will be the appropriate response when it occurs as a subsequent target. It is therefore possible that the presence of the letter $B$ in the prime produces interference in the form of response competition and that control mechanisms reduce this interference by inhibiting this response. Hence, response inhibition may play a role in producing NP. This observation receives empirical and theoretical support from the fact that the neural network model of Houghton and Tipper (1994) has been extended to response inhibition in the context of reaching movements and control of fixation in the presence of distracting targets (Houghton \& Tipper, 1999; Tipper, Howard, \& Houghton, 2000). In addition, Bowman et al. (2006) have recently extended the model to response inhibition in subliminal priming. A key aspect of this theory that we would draw attention to here is that inhibition of nontarget or interfering activations is "reactive"; that is, inhibition specifically directed at an activated node (or network of nodes) is generated locally by an inhibitory feedback loop from the node onto itself. Thus, the more active a node is, the more strongly it drives the inhibitory feedback system (Houghton, Tipper, Weaver, \& Shore, 1996).

One of the main reasons for the neglect of response factors in NP is the general belief that response inhibition is not necessary to observe it. Support for this comes from studies showing that NP survives changes in the modality of response. In these (few) studies, responses to the prime displays are typically given orally, using a voice key, and responses to the probe display are performed manually on the keyboard (Tipper, MacQueen, \& Brehaut, 1988). The finding of intact NP following a switch of response modality between prime and probe suggests that NP is not exclusively due to the inhibition of a motor response. However, these experiments have involved trial-by-trial task switching, and participants might have maintained both task sets (oral and manual responding) at a high level of activation on all trials. In this case, even though only an oral response was required on a prime trial, it is possible that the manual response was also being activated. In the process of selecting the correct response, all activated responses to the distractor were inhibited.

A better source of support for the idea that response inhibition is not necessary to observe NP comes from experiments with, for example, matching tasks in which distractors do not activate incompatible responses or with lexical decision tasks in which target and distractor are both words (Marí-Beffa, Fuentes, Catena, \& Houghton, 2000; Marí-Beffa, Houghton, Estévez, \& Fuentes, 2000). However, from the observation that NP occurs in the absence of response competition in the prime, one cannot logically conclude that when such competition is present, it is not involved in the effect. In this article, we present evidence that (1) "conventional" NP from ignored distractors does not have a single cause and (2) one cause is not perceptual at all but involves response selection. Before describing the logic of the experiments in more detail, we first take a closer look at some common NP designs to illustrate our view that a role for response inhibition is quite plausible and that the possibility has not been controlled for.

\section{On the Locus of Negative Priming: Is There a Role for Response Inhibition?}

It is now well established, using both reaction time (RT) and psychophysiological measures, that nontarget, distractor stimuli can evoke perceptual activity and motor responses (Coles, Gratton, Bashore, C. W. Eriksen, \& Donchin, 1985; De Jong et al., 1994; DeSoto et al., 2001; Eimer \& Schlaghecken, 1998; C. W. Eriksen, Coles, Morris, \& O’Hara, 1985; Heil, Osman, Wiegelmann, Rolke, \& Hennighausen, 2000; Praamstra \& Plat, 2001). However, if only correct responses are to be produced, the attentional control of action must involve the resolution of response competition caused by distractors. The neural locus of this form of control is generally attributed to the prefrontal lobes and is widely believed to involve the inhibition of contextually inappropriate responses (De Jong et al., 1994; Eimer, 1999; Hazeltine, Poldrack, \& Gabrieli, 2000; Heil et al., 2000; Jonides, Smith, Marshuetz, Koeppe, \& Reuter-Lorenz, 1998; Pliszka, Liotti, \& Woldorff, 2000; Shiu \& Kornblum, 1996). Interestingly, Metzler and Parkin (2000) report a loss of NP in patients with prefrontal lobe damage.

In most NP designs, the distractor in the prime trial is associated with a response different from that to the target and hence may give rise to response competition (Houghton \& Tipper, 1994). If this competition is controlled by response inhibition, then it could produce an inhibitory priming effect, if the suppressed response has to be quickly reactivated (see Eimer, 1999, and Eimer \& Schlaghecken, 1998, for both behavioral and ERP evidence of just such an effect). Few, if any, NP studies control for the confound with response selection, despite the fact that it is present in the most common procedures, including those used with special populations (Hasher, Stoltzfus, Zacks, \& Rypma, 1991; Kane, Hasher, Stoltzfus, Zacks, \& Connelly, 1994; Metzler \& Parkin, 2000; Tipper, 1991). Here, we discuss two specific NP paradigms to make this point clearer: location-based NP and Stroop NP (see Fox, 1995, and Tipper, 2001, for more detailed reviews and descriptions of various procedures). 
Location-based NP. In a location-based NP design, two objects are presented in different locations. The target is selected on the basis of a particular (nonspatial) attribute (e.g., identity), and the task is to determine its location (usually one of four well-marked locations) by moving a joystick in the direction of the target or pressing a key that is spatially compatible with the location. If, following a prime trial, the target object next appears in the location previously occupied by the distractor, RTs are slowed, relative to those to targets appearing at a previously empty location (Tipper, Brehaut, \& Driver, 1990; Tipper, Weaver, Cameron, Brehaut, \& Bastedo, 1991; Tipper, Weaver, Kirkpatrick, \& Lewis, 1991).

This effect has been commonly interpreted in perceptual terms, including inhibition of representations of distractor location (Tipper et al., 1990). However, the use of a small response set (four locations, repeatedly) and a highly compatible and fairly natural stimulus-response (S-R) mapping is likely to produce a high degree of response activation to the distractor, leading to a need for strong response inhibition. It is possible that this is responsible for some of the distinctive features of location NP, such as its persistence (Tipper, Weaver, Cameron, et al., 1991), robustness (Neill, Terry, \& Valdes, 1994) and superior effect size (Tipper et al., 1994). As far as we are aware, no location NP studies have fully removed this confound.

A response-inhibition account of localization NP is also compatible with recent findings regarding the role of distractor inhibition in controlling reaching movements (Baylis \& Baylis, 2001; Tipper et al., 2000; Tipper, Howard, \& Jackson, 1997). In this work, it has been argued that reaching (and eye fixation) trajectories to target objects can be influenced by the suppression of the parameters of activated responses to distractor objects (this form of response inhibition has been modeled computationally; Houghton \& Tipper, 1999; Tipper et al., 2000). In addition, if a spatial target is associated with a spatially incompatible response, it appears that the compatible response is activated and consequently suppressed, producing a response-based NP effect from a target object (Read \& Proctor, 2004).

Stroop NP. In the standard Stroop task, participants have to report the color of the ink in which an incongruent color word is displayed (e.g., the word red written in green). Stroop NP is found when, on a given trial, the ink color to be reported corresponds to the incongruent word on the previous trial (RTs increase relative to naming a new color). This is a popular and robust procedure, and the results have been used to argue for the inhibition of more central representations (Claridge, Clark, \& Beech, 1992; Dalrymple-Alford \& Budayr, 1966; Lowe, 1979, 1985; Marí-Beffa, Estévez, \& Danziger, 2000; Neill, 1977; Neill \& Westberry, 1987). However, the tendency to say the name of the prime color word is obviously strong and must be suppressed to name the ink instead. In support of this, the Stroop effect is much greater when a strongly associated response (i.e., naming the ink color) is used, relative to a novel response, such as a manual keypress (MacLeod, 1991). This response competition is frequently held to be the basis of Stroop interference (and related phenomena, such as the spatial Stroop effect; DeSoto et al., 2001). Furthermore, the incongruent (conflict) condition has been found to selectively activate areas of the prefrontal lobes associated with response suppression (Banich et al., 2000; Leung, Skudlarski, Gatenby, Peterson, \& Gore, 2000). Hence, it is quite possible that Stroop NP arises not from the inhibition of input lexical (or semantic) representations but from the suppression of the responses evoked by them.

In conclusion, NP in all these studies may be due (wholly or partly) to response inhibition rather than to processes involving the effect of selective attention on perceptual processing and/or perceptual memory traces. This possibility has serious implications for the use of NP as a tool to study cognitive control. Although authors do not in general favor (or even consider) explanations in terms of response suppression, it is clear that most NP studies do not in fact control for the possibility. In this article, we present a design in which, for the first time, NP due to the mere repetition of an ignored stimulus (without response competition) is dissociated from NP tied to response factors. Henceforth, we will refer to these two forms of negative priming as object $N P$ and response $N P$, respectively. For clarity, we will generally refer to the combination of the two forms as conventional NP.

\section{The Present Study}

To show that response level processes are not producing NP in studies using incompatible distractors, it is not sufficient to find some cases in which NP is still observed even though the potential involvement of response suppression is minimized. Instead, the perceptual and response aspects of the prime distractor must be dissociated in the probe trial. If a probe target is associated with the same response as the prime distractor but is nevertheless perceptually distinct from it, then no NP should be observed, if the effect is entirely due to perception and/or perceptual memory. On the other hand, if response suppression is involved, then the requirement to produce the same response to a probe target as would have been activated by a probe distractor should result in response NP, even when the prime distractor and probe target are perceptually distinct. This is the logic of the following experiments.

One of the properties of conventional NP that has been commonly explained in perceptual terms is its relationship with practice. Thus, it has been found that NP increases with practice (Strayer \& Grison, 1999) and does not disappear with more extensive practice (Tipper, Eissenberg, \& Weaver, 1992). More specifically, Strayer and Grison show that for a distractor to produce NP, it must first be experienced a number of times as a target. But again, the designs used in these studies confound perceptual and response factors in NP. According to our analysis, conventional NP has not been found to disappear with extensive practice because response-incompatible distractors have 
been used. The logic behind this reasoning is as follows and is based on the reactive inhibition model of Houghton and Tipper (1994).

In a typical experimental session, participants are asked to respond to attended stimuli in a novel way (e.g., by pressing keys or moving a joystick). Consequently, they first perform a block of practice trials, in which these unusual S-R mappings are consolidated (the responses collected are discarded). Although participants should reach a reasonable degree of proficiency by the time the data proper are collected, S-R learning may continue throughout the experiment. As the S-R mappings become consolidated, responses to stimuli should be activated more rapidly and automatically, without participants having to think about the mapping rule they have been instructed to follow. This should lead to distractors becoming more "distracting" at response levels and, consequently, to a need for greater response inhibition. This proposal leads to the prediction that response NP will tend to increase with practice.

On the other hand, continuous practice with the same stimuli appears to lead to perceptual habituation to otherwise salient distractors (see Bruin, Kenemans, Verbaten, \& Van der Heijden, 2000, for an ERP study showing progressive habituation of sensory-evoked potentials caused by distractor stimuli). According to the reactive inhibition model of Houghton and Tipper (1994), this should lead to a reduction in object NP (Houghton et al., 1996). Hence, in direct contrast to response NP, we predict that object $N P$ will tend to decrease with practice.

According to this analysis, conventional NP has not been found to disappear with extensive practice because response-incompatible distractors have been used. We tried to replicate this basic finding in Experiment 1. But in addition, contrary to previous results, we predicted that NP would attenuate with practice if compatible distractors were used, as a result of perceptual habituation. We tested this in Experiment 2.

\section{EXPERIMENT 1}

Responses to a target stimulus are affected by the relationship it has with any copresent distractors (Duncan \& Humphreys, 1989). Interference effects result not only from perceptual relatedness but also from the compatibility/ incompatibility of the response categories associated with target and distractors (B. A. Eriksen \& C. W. Eriksen, 1974; C. W. Eriksen et al., 1985; Heil et al., 2000; Miller, 1991). In the present experiment, we assessed whether the response compatibility of prime targets and prime distractors is important to produce NP. To do so, we use a design based on the flanker task (B. A. Eriksen \& C. W. Eriksen, 1974), in which a central target stimulus is flanked on either side by a distractor. In our design, the distractor stimuli were never the same as the targets; however, they could have been, in the context of the experiment, associated with a competing response (incompatible condition) or not associated with any response (neutral condition). In this way, we hoped to disentangle effects due simply to the repetition of an ignored stimulus from those that might have been due to the need to activate a recently suppressed response. The flanker paradigm was specifically chosen since it is known to produce a robust difference in degree of interference between response-neutral and responseincompatible distractors. Furthermore, psychophysiological studies have provided compelling evidence that this is largely due to response competition in the incompatible condition (Coles et al., 1985; Heil et al., 2000). Finally, in both fMRI and ERP studies, the incompatible condition has been found to selectively elicit prefrontal lobe activation associated with response suppression (Hazeltine et al., 2000; Heil et al., 2000).

In addition, we expected to find that NP does not disappear with practice (Strayer \& Grison, 1999; Tipper et al., 1992). We believe that NP in these previous studies resists practice because the type of NP under study emerged from the inhibition of the incompatible response. Since practice helps to strengthen the mapping between the stimuli and their responses, the need for inhibition would only increase with practice but would not be reduced.

\section{Method}

Participants. Twenty-three participants from the University of Wales participated in this experiment. All of them received course credits for their participation. None of them reported any visual or reading impairment.

Apparatus and Stimuli. The stimuli used in the experiment were the digits $1-8$, to which the participants responded by pressing one of four keys on the keyboard ("C," "V," "N," and "M"). Each numeral was $5 \mathrm{~mm}$ high and $4 \mathrm{~mm}$ wide $\left(0.48^{\circ}\right.$ and $0.38^{\circ}$ of visual angle, respectively). The target was displayed at fixation while two identical distractors were displaced from fixation at $0.95^{\circ}$ of visual angle $(1 \mathrm{~cm})$ to the right and the left of the target. Stimuli were displayed in black on a white background, on an IBM-compatible PC, and responses were made on a standard keyboard.

Design and Procedure. Each trial began with an asterisk (*) in the center of the screen, which remained for $500 \mathrm{msec}$. After an interval of $150 \mathrm{msec}$, the prime display (D1) was presented until response, and $300 \mathrm{msec}$ later, the probe display (D2) was displayed until response or until the end of a 2,000-msec waiting window. Trials with no response were counted as errors. The intertrial interval was fixed at $300 \mathrm{msec}$. Each possible target was repeated the same number of times.

All the participants were required to respond to the target numbers 1 and 2 by pressing the "C" key, to the numbers 3 and 4 by pressing the "V" key, to the numbers 5 and 6 by pressing the "N" key, and to the numbers 7 and 8 by pressing the "M" key (Figure 1). All the displays were incompatible; that is, target and distractors always belonged to different response categories (e.g., 3 and 6).

The different priming conditions were built on the relationship between the D1 (prime) distractor and the D2 (probe) target. Examples of the conditions are shown in Figure 1. The three conditionscontrol, related, and repeated - were constructed as follows: In the control condition, all four stimuli (target and distractor from D1 and D2) were different and belonged to different responses (e.g., 757 followed by 424 ). The related condition was identical to the control condition, except that the D1 distractor and the D2 target, while still different stimuli, were associated with the same response (e.g., 151 followed by 424 ). In the repeated condition, the D1 distractor and the D2 target were identical (e.g., 252 followed by 424 ). Note that our repeated condition was equivalent to the conventional NP condition, the prime distractor being incompatible with the prime target and repeated as the probe target. 


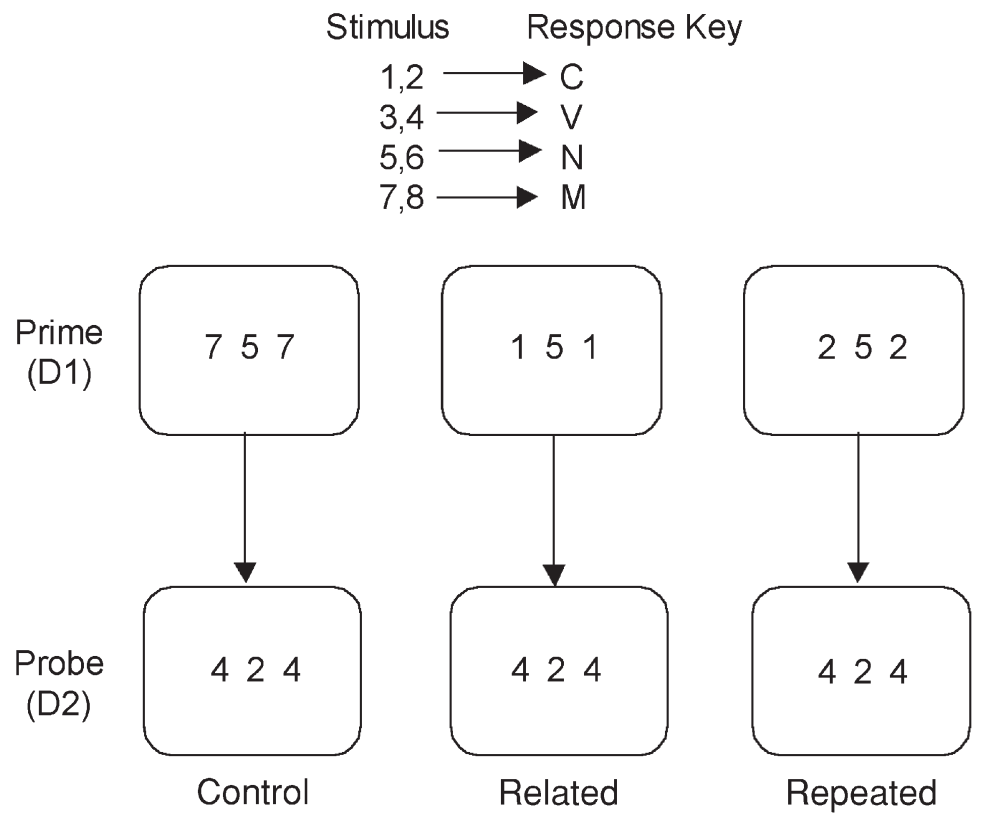

Figure 1. The three conditions in Experiment 1, showing example primeprobe pairs. The stimulus-response mapping is shown at the top, and the target is always the central digit. Flanker (distractor) digits were always response incompatible with the target. In the control condition, there was no relation between any of the items in the prime and the probe. In the related condition, the probe target shared a response with the prime distractor but was a distinct stimulus. In the repeated condition, the prime distractor and the probe target were the same. The repeated condition was an example of a conventional negative priming trial.

Response NP was measured by comparing the results of the control condition with those of the related condition. Inclusion of the conventional NP measure (control minus repeated) gave us an index of the size of the normal NP effect with this paradigm and permitted us to assess any difference due to also ignoring the incompatible object at a perceptual level.

Another 12 filler trials were included to balance the repetition of items and the combination of target and distractors across all the displays. As a result, every item was presented the same number of times and was combined with every other stimulus in both first and second displays. As a result, there were 336 trials overall, separated in seven blocks of 48 trials. Each block contained 12 trials per critical condition (control, related, and repeated) and 12 fillers. The order of the trials within each block, practice and experimental, was individually randomized for each participant.

The participants performed a few trials in the presence of the experimenter to demonstrate understanding of the instructions. Also, the instructions put a special emphasis on accuracy, given the relatively low number of observations per condition and per block.

\section{Results}

Mean RTs and standard deviations to D2 displays per block and condition are presented in Table 1. Only correct responses (in both D1 and D2) were analyzed. The means of median RTs to the probe display per participant, condi-

Table 1

Means of Median Probe Reaction Times (RTs, in Milliseconds) and Standard Deviations (SDs) per Condition and Block of Trials in Experiment 1

\begin{tabular}{|c|c|c|c|c|c|c|c|c|c|c|c|c|c|c|}
\hline & \multicolumn{14}{|c|}{ Block } \\
\hline & \multicolumn{2}{|c|}{1} & \multicolumn{2}{|c|}{2} & \multicolumn{2}{|c|}{3} & \multicolumn{2}{|c|}{4} & \multicolumn{2}{|c|}{5} & \multicolumn{2}{|c|}{6} & \multicolumn{2}{|c|}{7} \\
\hline & RT & $S D$ & RT & $S D$ & RT & $S D$ & RT & $S D$ & RT & $S D$ & RT & $S D$ & RT & $S D$ \\
\hline Repeated condition & 739 & 131 & 683 & 110 & 629 & 81 & 648 & 97 & 637 & 108 & 655 & 106 & 637 & 105 \\
\hline Related condition & 713 & 159 & 663 & 122 & 653 & 107 & 627 & 106 & 603 & 97 & 638 & 131 & 635 & 104 \\
\hline Control condition & 733 & 145 & 665 & 125 & 634 & 81 & 610 & 88 & 609 & 95 & 601 & 92 & 597 & 110 \\
\hline \multicolumn{15}{|l|}{ Negative priming } \\
\hline Object + response & -6 & & -18 & & 5 & & $-38^{*}$ & & $-28^{*}$ & & $-54^{*}$ & & $-40^{*}$ & \\
\hline Response & 20 & & 2 & & -19 & & -17 & & 6 & & $-37^{*}$ & & $-38^{*}$ & \\
\hline
\end{tabular}

Note-Object + response negative priming refers to the RTs in the control condition minus those in the repeated condition; response negative priming refers to the RTs in the control condition minus those in the related condition. ${ }^{*} p<.05$. 


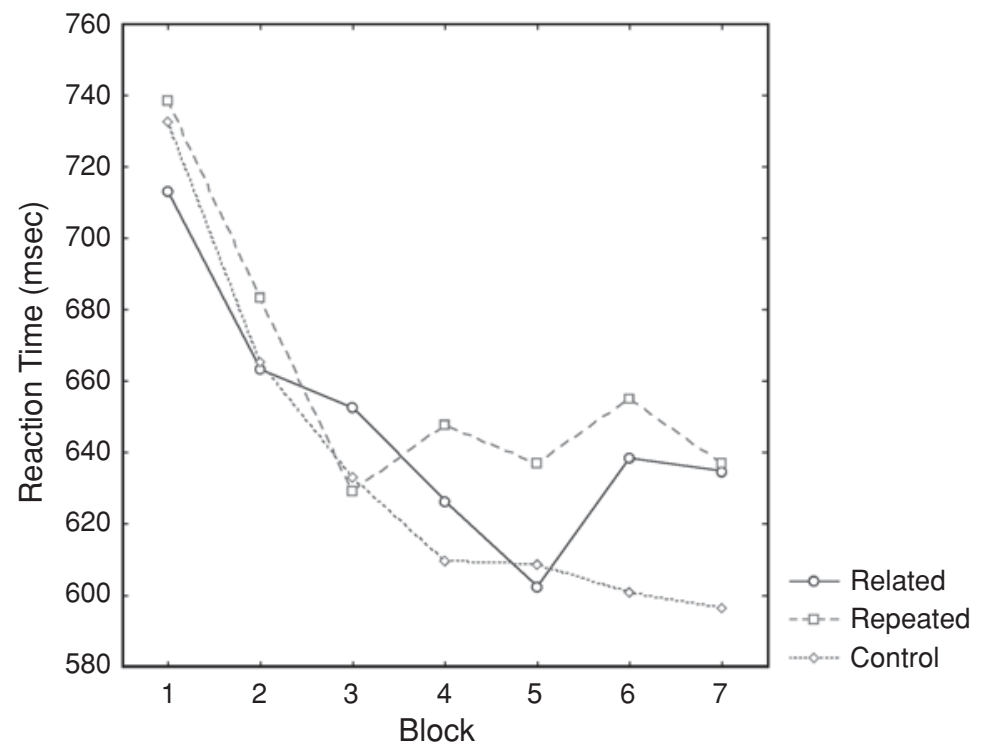

Figure 2. Results from Experiment 1. Mean reaction times from probe trials. Significant negative priming (NP) emerged only in the two experimental conditions after some practice. It appeared in Block 4 for the repeated condition (object + response NP) and not until Block 6 for the related condition (response NP).

tion, and block were analyzed using a 3 (conditions) $\times 7$ (blocks) repeated measures ANOVA.

There was a significant main effect of conditions $\left[F(2,44)=7.97, M S_{\mathrm{e}}=3,360.32, p<.002\right]$. There was a significant global effect of conventional NP (control vs. repeated) $\left[F(1,22)=40.64, M S_{\mathrm{e}}=1,316.48, p<.0001\right]$ but not of response NP (control vs. related) $[F(1,22)=$ $2.5, p=.13$ ]. In addition, $\mathrm{RT}$ s in the repeated condition were marginally slower than those in the related condition $\left[F(1,22)=3.69, M S_{\mathrm{e}}=4,154.26, p=.068\right]$.

There was a significant main effect of practice $\left[F(6,132)=10.34, M S_{\mathrm{e}}=10,376.48, p<.00001\right]$. In the trend analysis, responses got linearly faster as the blocks of trials progressed $\left[F(1,22)=17.34, M S_{\mathrm{e}}=24,609.8, p<\right.$ .0005]. Most importantly, the differences between conditions significantly changed with practice $[F(12,264)=$ $\left.2.1, M S_{\mathrm{e}}=2,321.8, p<.02\right]$. We ran separate tests to analyze conventional NP (control vs. repeated) and response NP (control vs. related) for each block of trials (see Figure 2). Conventional NP was significant from Block 4 to Block 7 [Block 4, $F(1,22)=8.7, p<.01$; Block 5, $F=10.22, p<.01$; Block $6, F=22.82, p<$ .0001 ; Block 7, $F=37.91, p<.00001]$. Response NP had a later onset and was significant only for Blocks 6 and 7 [Block 6, $F(1,22)=6.33, p<.02$; Block $7, F=20.66$, $p<.0002]$. We also analyzed the differences between the results in the related condition and those in the repeated condition across the blocks. RTs in the repeated condition were significantly slower than those in the related condition only in Block $5[F(1,22)=6.94, p<.02]$. There were no other reliable differences.

\section{Discussion}

The main purpose of Experiment 1 was to investigate whether NP can result from the incompatible response associated with a prime distractor, without repetition of the distractor itself. In addition, we wished to track the development of NP as a function of experience with a particular set of novel S-R mappings. We hypothesized that, as the S-R mapping became consolidated through practice, the efficiency with which a flanker stimulus would activate its associated response would improve. According to a reactive model for the inhibitory control of interference (Houghton \& Tipper, 1994), this would lead to greater inhibition of response codes. Hence, response NP should take time to emerge but not disappear with further practice (cf. Strayer \& Grison, 1999; Tipper et al., 1992).

The results confirmed both the existence of response NP and its predicted time course. NP in the related condition appeared after considerable practice, from Block 6, and then showed no signs of reduction. The inclusion of a conventional NP condition (control vs. repeated) in the experiment allowed us to compare it to response NP, and to thereby assess any contribution from the repetition of the prime distractor as target. There was some evidence that conventional NP was indeed more robust than response NP. RTs in the repeated condition were globally slower than those in the related condition, and NP from the repeated condition started to emerge earlier (Block 4) than that from the related condition (Block 6). Hence, conventional NP also requires some practice with the stimulus objects before it appears. This is to be expected if response NP is a component of conventional NP. In ad- 


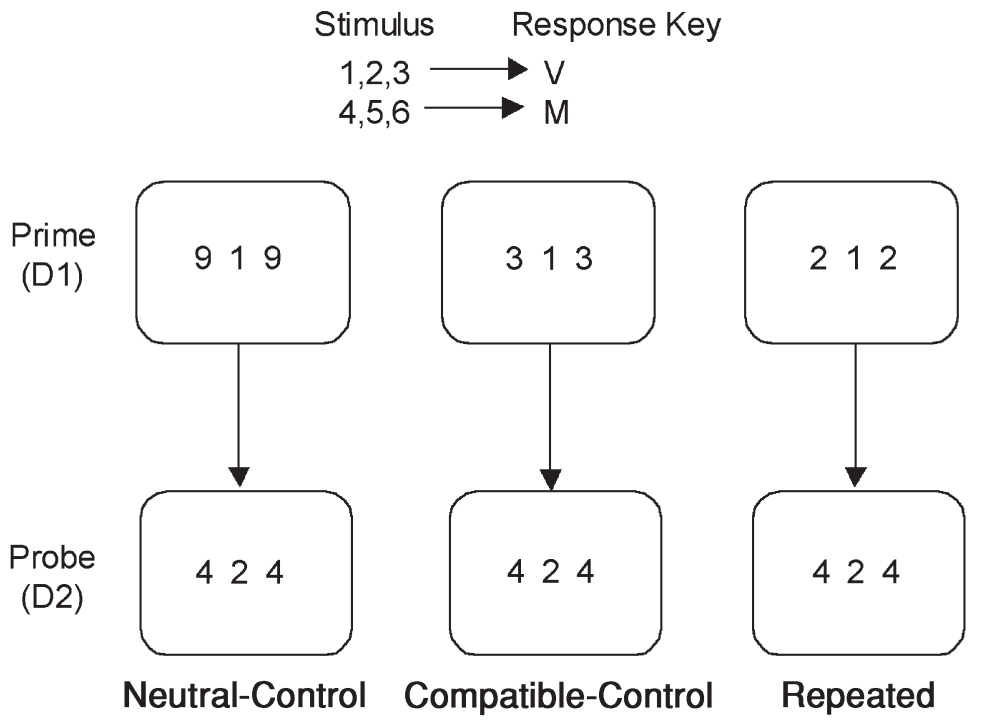

Figure 3. The three conditions in Experiment 2, showing example primeprobe pairs. The stimulus-response mapping is shown at the top. The target was always the central digit. In the neutral-control condition, there was no relation between any of the items in the prime and probe, and the prime distractors were response neutral. In the compatible-control condition, the probe target shared a response with the prime distractor but was a distinct stimulus. In the repeated condition, the prime distractor and the probe target were the same. The repeated condition was similar to the repeated condition in Experiment 1 (Figure 1), but the prime distractor was compatible with the response to the target.

dition, Strayer and Grison (1999) suggested that it is necessary that the experimental stimuli increase their level of activation in memory in order to produce NP. Our results are compatible with this view, but they further suggest (given the late emergence of response NP) that the consolidation of $\mathrm{S}-\mathrm{R}$ mappings is important for observing NP with repeated objects.

In Experiment 1, we used only incompatible distractors and hence did not directly address the issue of the contribution of object NP to conventional NP or of its relation to practice. (Indeed, given that the vast majority of studies on NP from ignored stimuli confound stimulus and response factors, there is very little data at all on NP entirely due to the re-presentation of a previously ignored stimulus that did not also compete for response in the prime.) The arguments of Strayer and Grison (1999) and the results of Experiment 1 indicate that pure object NP will also build up over time. However, as argued earlier, it is also possible that participants may eventually start to habituate to frequently encountered distractors. If this happens then, according to a reactive inhibition model, object NP should eventually start to diminish. The disappearance of NP with practice has not been reported hitherto; hence, the next experiment was designed to test whether object NP may disappear with practice.

\section{EXPERIMENT 2}

The goal of Experiment 2 was to examine the effect of practice on object NP. To do so, we needed to remove any possible contribution from response NP. We assessed NP from prime distractors that did not compete with the target for response (i.e., were compatible). We used two control conditions: one condition in which the distractors were not associated to any response and hence never appeared as targets (neutral), and another condition in which the prime distractors were associated with a compatible response, but all the stimuli in prime and probe displays were different. The last condition was used to test any form of response NP following compatible primes.

\section{Method}

Participants. There were twenty-two participants in this experiment. All were students from the University of Wales, who received extra course credits for their participation. None reported any visual or reading impairment.

Apparatus and Stimuli. Stimuli and materials followed those used in the previous experiment. This time we used the digits 1-9. The digits 1-6 were to be responded to by pressing one of two keys on the keyboard ("V" and "M"). The numbers 7-9 were used as neutral distractors.

Design and Procedure. The sequence of events and timing operation replicated those used in the previous experiment. Only two response alternatives were used this time. Thus, the participants had to respond to the targets 1,2 , and 3 by pressing the "V" key on the keyboard and to the targets 4,5 , and 6 by pressing the " $\mathrm{M}$ " key (Figure 3). Neutral distractors were used only in the neutral-control condition, and the items in D2 (probe) were always incompatible. Thus, the experimental conditions were as follows (Figure 3 ): In the neutral-control condition, the D1 distractors were neutral, none of the D1 or D2 stimuli were related, and the responses to perform toward D1 and D2 targets were the same (e.g., 919 followed by 4 $24)$. The compatible-control condition was identical to the neutral- 


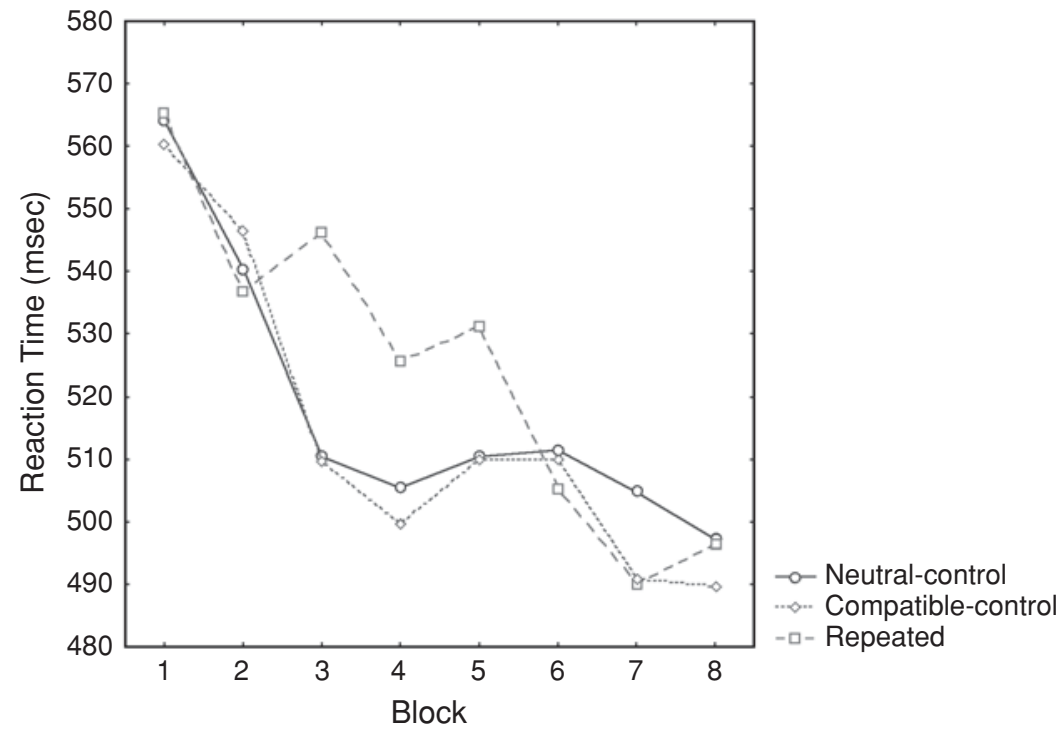

Figure 4. Object negative priming (NP) in Experiment 2. Mean reaction times from probe trials. The two control conditions did not differ. Object NP emerged only in the middle blocks, disappearing with further practice.

control condition, except that the D1 distractors were compatible with the response to the target, and the distractor in D1 belonged to the same response category as the D2 target (e.g., 313 followed by 4 $24)$. The repeated condition was identical to the compatible-control condition, except that the prime distractor and the probe target were the same (e.g., 212 followed by 424 ).

NP from a repeated compatible distractor could now be measured with respect to two different control conditions. The neutralcontrol condition provided a case in which the D1 distractors were not associated with any response. Perhaps more interestingly, in the compatible-control condition, the D1 distractors were compatible and therefore could provide a better comparison with the repeated condition. However, it is important to note that we did not actually predict any difference between the two control conditions, since no inhibition should be directed to the response associated with the compatible distractor.

Experiment 1 used all incompatible distractors, which we supposed would cause interference in responding to the target. However, we were unable to measure this, since the experiment contained no neutral or compatible distractors to which a comparison could be made. We remedied this in Experiment 2 by using compatible and neutral distractors in the prime but incompatible ones in the probe. This permitted us to assess interference effects from incompatible distractors and to track them as a function of practice.

In this experiment, there were 12 trials of each experimental condition in each of eight different blocks of trials. Also, given that all these trials were based on conditions in which D1 and D2 responses were the same, we also included 24 filler trials in which responses changed from D1 to D2. As in Experiment 1, every item was presented the same number of times and was combined with every other stimulus in both first and second displays. There were a total of 480 trials separated in eight blocks.

Instructions and delivery conditions were the same used in Experiment 1 .

\section{Results}

Negative priming effects. The RTs and accuracy were processed following the same procedure used in Experiment 1 . The results are shown in Figure 4.
Responses to the probe display were analyzed following a 3 (conditions) $\times 8$ (blocks) repeated measures ANOVA. Results showed a significant effect of practice (block) $\left[F(7,147)=9.17, M S_{\mathrm{e}}=3,912.04, p<.00001\right]$, since overall responses linearly decreased over time $[F(1,21)=$ $\left.31.65, M S_{\mathrm{e}}=6,860.7, p<.00001\right]$. There was also a main effect of the experimental conditions $[F(2,42)=$ 5.72, $\left.M S_{\mathrm{e}}=786.67, p<.01\right]$, due to significant NP in the repeated condition with respect to both control conditions [repeated vs. neutral-control, $F(1,21)=4.95$, $M S_{\mathrm{e}}=756.79, p<.005$; repeated vs. compatible-control $\left.F(1,21)=11.04, M S_{\mathrm{e}}=789.29, p<.005\right]$. There were no significant differences between the two control conditions. Importantly, there was a significant interaction between blocks and conditions $\left[F(14,294)=1.74, M S_{\mathrm{e}}=\right.$ $1,240.89, p<.05]$.

We further analyzed separately the effect of practice on the two different measures of NP (Table 2). As predicted, object NP took time to emerge and disappeared with extended practice. Object NP measured with respect to the neutral-control condition was significant only from Block 3 to Block 5 (Block $3, F=12.46, p<$ .002 ; Block 4, $F=4.44, p<.05$; Block 5, $F=4.63, p<$ $.05)$. When object NP was measured with respect to the compatible-control condition, the result was the same (Block 3, $F=11.99, p<.005$; Block 4, $F=12.32, p<$ .005 ; Block 5, $F=6.02, p<.05)$.

The two control conditions showed no significant differences at any level. The error rates showed no differences.

Interference effects. Given the characteristics of this design, it was possible to analyze the changes in flanker interference across blocks. The responses to all compatible primes were collapsed per block to produce a new compatible condition. Collapsing all neutral primes created a neutral condition. From the incompatible probes, 
Table 2

Means of Median Probe Reaction Times (RTs, in Milliseconds) and Standard Deviations (SDs) per Condition and Block of Trials in Experiment 2

\begin{tabular}{|c|c|c|c|c|c|c|c|c|c|c|c|c|c|c|c|c|}
\hline & \multicolumn{16}{|c|}{ Block } \\
\hline & \multicolumn{2}{|c|}{1} & \multicolumn{2}{|c|}{2} & \multicolumn{2}{|c|}{3} & \multicolumn{2}{|c|}{4} & \multicolumn{2}{|c|}{5} & \multicolumn{2}{|c|}{6} & \multicolumn{2}{|c|}{7} & \multicolumn{2}{|c|}{8} \\
\hline & RT & $S D$ & RT & $S D$ & RT & $S D$ & RT & $S D$ & RT & $S D$ & $\mathrm{RT}$ & $S D$ & RT & $S D$ & RT & $S D$ \\
\hline Repeated condition & 565 & 67 & 537 & 51 & 546 & 77 & 525 & 84 & 531 & 59 & 505 & 67 & 490 & 64 & 497 & 64 \\
\hline Compatible-control condition & 560 & 67 & 547 & 72 & 510 & 61 & 500 & 69 & 510 & 70 & 510 & 71 & 491 & 57 & 490 & 64 \\
\hline Neutral-control condition & 564 & 68 & 540 & 57 & 511 & 55 & 505 & 67 & 511 & 69 & 512 & 70 & 505 & 67 & 497 & 72 \\
\hline \multicolumn{17}{|l|}{ Object negative priming } \\
\hline Neutral-control minus repeated & -1 & & 3 & & $-35^{*}$ & & $-20^{*}$ & & $-20^{*}$ & & 7 & & 15 & & 0 & \\
\hline Compatible-control minus repeated & -5 & & 10 & & $-36^{*}$ & & $-25^{*}$ & & $-21^{*}$ & & 5 & & 1 & & -7 & \\
\hline
\end{tabular}

${ }^{*} p<.05$.

we created a new incompatible condition. The results are shown in Figure 5.

The mean of median RTs for correct trials in these conditions were submitted to a 3 (conditions: incompatible, compatible, neutral) $\times 8$ (blocks) repeated measures ANOVA. Results showed a significant main effect of practice $\left[F(7,147)=6.7, M S_{\mathrm{e}}=3,712.67, p<.0001\right]$, where the trend analysis demonstrated a linear global decrease in RTs $\left[F(1,21)=13.57, M S_{\mathrm{e}}=8,838.0, p<.002\right]$. There was also a main effect of flanker interference $[F(2,42)=$ $\left.19.61, M S_{\mathrm{e}}=1,468.58, p<.0001\right]$. Responses in the incompatible condition were slower than those in both the compatible condition $\left[F(1,21)=52.72, M S_{\mathrm{e}}=1,066.96\right.$, $p<.0001]$ and the neutral condition $[F(1,21)=13.09$, $\left.M S_{\mathrm{e}}=1,727.32, p<.002\right]$. Significant facilitation was observed from compatible distractors relative to neutral distractors $\left[F(1,21)=4.68, M S_{\mathrm{e}}=1,611.46, p<.05\right]$. This supports our view that inhibition is not directed toward responses activated by compatible distractors.
The interaction between conditions and blocks did not produce a significant result. Given the large number of blocks, we did not expect a significant interaction; nevertheless, we looked at the topography of the different types of flanker interference through the blocks (see Figure 5). Flanker interference in incompatible displays took time to emerge; when compared with the neutral trials, a significant slowing of RTs in incompatible trials was found from Block 4 to Block 8 (Block 4, $F=15.85, p<.001$; Block 5, $F=6.55, p<.02$; Block 6, $F=4.84, p<.05$; Block 7, $F=7.96, p<.02$; Block $8, F=8.17, p<.01$ ). A similar result was obtained comparing incompatible trials with compatible ones (Block $4, F=7.78, p<.02$; Block 5, $F=10.60, p<.005$; Block $6, F=6.00, p<.05$; Block 7, $F=5.54, p<.05$; Block $8, F=15.19, p<.001$ ). This latter effect also reached significance in the first block $(F=4.80, p<.05)$. This is explained by facilitation from compatible flankers (neutral vs. compatible trials), which appeared only during the first two blocks of trials (Block 1,

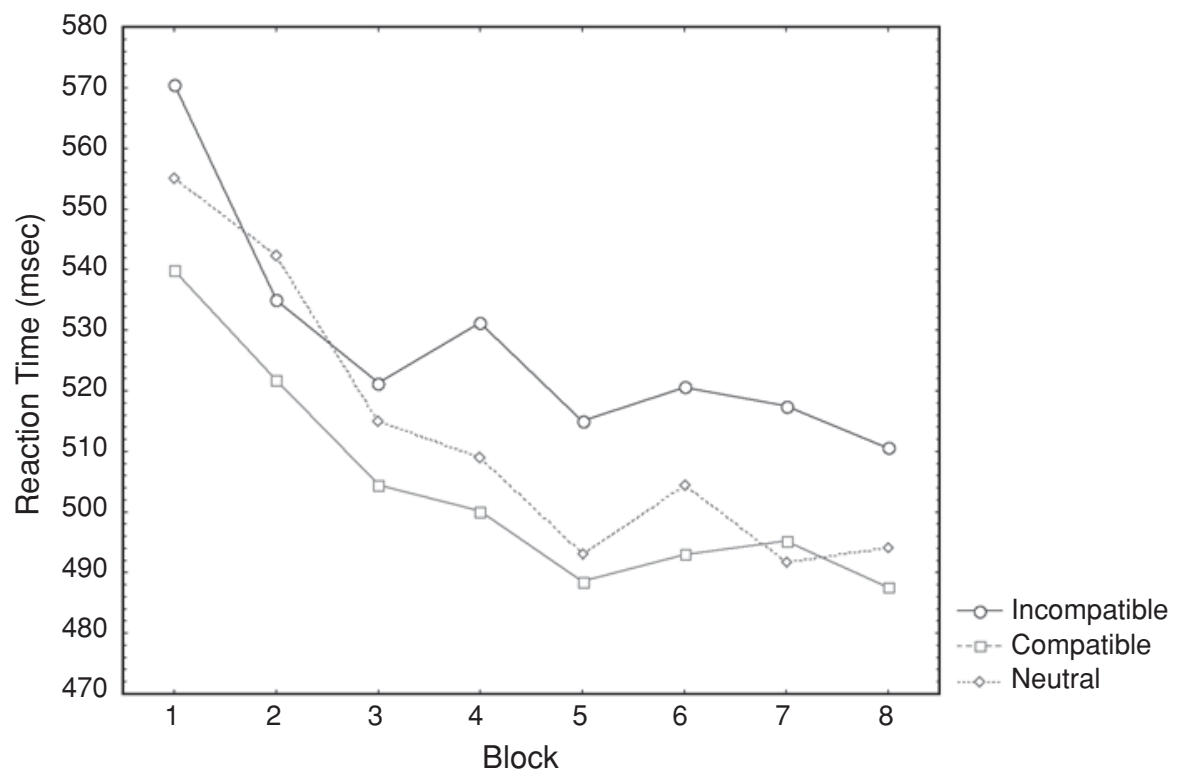

Figure 5. Interference as a function of practice in Experiment 2. Interference was measured as the difference in reaction times between incompatible and neutral trials. 
$F=5.17, p<.05$; Block 2, $F=6.99, p<.02)$. The error rates did not show significant differences.

\section{Discussion}

The results of this experiment are new, showing that object NP took some time to emerge and then disappeared with further practice. Because all the items in the prime displays were response compatible for the critical conditions, the results can only be accounted for with reference to the perceptual properties of the distractor object and not to the response associated to it.

The results for object NP are in agreement with what was found in Experiment 1 for conventional NP - that some repetition of the stimuli is required before any NP begins to be observed. As we mentioned earlier, Strayer and Grison (1999) suggested that distractor objects need to increase their level of activation in memory to produce NP. However, in their design, all the distractors were associated to incompatible responses; thus, it was not clear whether this initial practice affected the memory representations of the objects or their associations with their corresponding responses. Our data demonstrate that practice does increase NP associated just to the perceptual properties of the distractor. In our framework, the repetition of these objects in the experimental context would help the sensitization of the systems engaged in their detection. This would result in the increased interference when they appear as distractors, requiring further inhibition to cope with them. As a result, NP increases with the repetition of the stimuli.

But with repeated exposure, these systems become habituated, and they stop responding to their perceptual features with the same intensity. Thus, object NP should disappear with extended practice, contrary to what was suggested by Tipper et al. (1992). Our data clearly show that if the distractors do not compete for the responses, NP disappears as they become less salient with practice.

The results on flanker interference derived from Experiment 2 support the above conclusions and also those drawn from Experiment 1. If our contention that response $\mathrm{NP}$ develops with the consolidation of S-R mappings is correct, then the time course of flanker interference and response NP should be similar, since the degree of interference can be taken as a measure of the activation of a response to the distractor. In a reactive model of response inhibition, it is the unwanted activation that "energizes" the inhibitory process (Bowman et al., 2006; Houghton \& Tipper, 1994). We found that reliable flanker interference did not emerge until Block 4 and then remained stable, similar to the pattern for response NP shown in Experiment 1.

In contrast, facilitation from compatible flankers (compatible vs. neutral conditions) was present in the early blocks (reaching significance in Blocks 1 and 2) and thereafter generally declined (Figure 5). Clearly, the relationship between this pattern and object NP is somewhat complex, since a significant compatibility effect preceded the onset of significant object NP. Nevertheless, the de- cline in facilitation with practice supports our view that the participants were habituating to the stimuli, and, in the final blocks, neither facilitation nor object NP was found.

A potential weakness of the present experiments is that the two forms of NP and the relationship between the flanker interference and response NP were measured in separate experiments. In the ideal case, all these measures would be taken in a single repeated measures design. Unfortunately, there are practical difficulties in doing so. To measure practice effects, we need a sufficient number of blocks for effects to emerge and possibly disappear, and all conditions must be measured equally within each block. To measure response NP with properly balanced conditions, at least four response alternatives are needed (Experiment 1). When measuring object NP, at least three stimuli must be mapped onto each response (Experiment 2). Thus, combining all the conditions from Experiments 1 and 2 in a single design requires 12 stimuli mapped onto four responses. Such a complex mapping would require considerable practice before the experiment began and numerous trials in each condition (of which there would be six). To circumvent this difficulty, we have run a reduced version with only two response alternatives and five blocks of trials; however, since this leads to some prime-probe contingencies, we do not include the experiment here. Nevertheless, the basic pattern of results in this combined design confirmed those reported here: Both conventional and response NP took time to emerge and were still present in the final (fifth) block; object NP, in contrast, emerged earlier, being at its maximum in the second block, but then faded, being completely absent in the final two blocks. Hence, the present evidence indicates that finding these starkly contrasting patterns of NP in relation to practice does not depend on running the relevant conditions in isolation.

\section{GENERAL DISCUSSION}

In this article, we have presented two experiments showing that (1) the resolution of response competition can, in itself, produce NP, (2) this is most likely a component of the effect in studies in which the ignored prime distractor, as well as being repeated in the probe, is associated with an incompatible response (i.e., in most standard NP paradigms), and (3) response NP is dissociable from object NP due simply to the repetition of an ignored distractor object as a target (without response incompatibility).

\section{On the Role of Response Competition in Negative Priming}

In this article, we have shown that response compatibility during the selection of the target in the prime display has a strong effect on subsequent NP. Incompatible responses associated with a distractor produce a subsequent cost in RTs, even when the distractor object is not repeated as a target (Experiment 1). Also, when both the response and the distractor are repeated (conventional NP), 
the NP effect seems to be more robust than that obtained from either response NP or object NP separately. Thus, in Experiment 1, conventional NP emerged earlier than response NP and, unlike object NP in Experiment 2, did not diminish with further practice. The results on flanker interference from Experiment 2 showed its time course to be similar to that of response NP and supported the idea that consolidation of S-R mappings through practice was causing responses to incompatible distractors to become automatically activated. Taken together, the results support a view of attentional control mechanisms as reactive (adaptive), sensitive to both the relative salience of target and distractor representations and the strength of their associated responses.

As noted earlier in this article, we chose a paradigm, the flanker task, which has been shown to induce electrophysiologically detectable response competition (Coles et al., 1985; Heil et al., 2000) and which activates prefrontal lobe selection mechanisms (Hazeltine et al., 2000). The results on interference from Experiment 2 showed that this response competition was taking place in these experiments. Hence, it is clear in this case that apart from selecting the correct stimulus, participants need to select the correct response, when inappropriate ones are activated. If this is also true of other common NP paradigms, as we argued earlier, then we expect that more accurate participants (those more successful at avoiding inappropriate responses) will show higher NP than will less accurate ones. Indeed, NP is enhanced when instructions emphasize accuracy rather than speed (Neill \& Westberry, 1987; Neumann \& DeSchepper, 1992; Nievas \& MaríBeffa, 2002). Also, this could explain why NP typically correlates positively with prime interference, at least in normal younger adults (Fox, 1994; Neill et al., 1992).

From a functional point of view, it should not matter whether interference is increased due to the manipulation of perceptual features or response competition. Any source of interference will affect the selection of the correct object and the correct response to it. We have shown here that certain properties of NP are more readily associated to response selection than to perceptual selection. These are dissociable processes with distinct characteristics that merit a separate analysis.

\section{The Effect of Practice on Negative Priming}

We believe that the dissociation between object NP and response NP through practice reveals the dynamic and reactive nature of inhibitory mechanisms of selection, which are modulated depending on the demands of the task. At the beginning of an experiment, the stimuli are rather novel and salient, and the participant needs to get practice with both their perceptual features, their relative locations, their identities, and their associated responses. It is during this phase that distractors are most salient (Bruin et al., 2000). Hence, participants need to make sure that they are attending to the correct stimulus (the target). If NP reflects the inhibition of distractors during an attentional visual selection process, then this effect is more likely to be observed associated to perceptual (or object-based) features during the first stages of the experiment. Once the participants are habituated to the stimuli, their relative locations, and probably the rhythm and timing of the task, distractors may achieve less perceptual activation, requiring less active inhibition.

In parallel, as practice progresses, stimuli become more and more automatically connected to their responses. One of the advantages of this process is that it enables the automatic activation of responses to targets. However, it brings an additional disadvantage: Distractors also activate these responses in parallel automatically. This decrease in perceptual interference but increase in response competition brings a qualitative change in the selection scenario. As practice increases, attentional selection needs to deal more with simultaneously activated responses, rather than simultaneously presented objects. We suggest that this is why NP from incompatible objects increases with practice.

The result showing that NP can disappear with practice (Experiment 2) may appear to contradict the recent proposals of Strayer, Grison, and colleagues (Grison \& Strayer, 2001; Malley \& Strayer, 1995; Strayer \& Grison, 1999). These researchers have shown that NP is contingent on stimulus repetition. For example, Strayer and Grison (1999) found NP from words that were repeated throughout the experiment but never from novel words. However, it is important to note that, in our experiments, we never tested NP from novel objects and also that the reduction we observed in object NP appears after extensive practice (more than 100 repetitions of each stimulus appearing as a target). Therefore, our data do not argue for or against the role of stimulus repetition in NP. Interestingly, Strayer, Grison, and colleagues argue that the repetition effects reveal an inhibitory mechanism of selection that is reactive to the level of activation of interfering responses and that works to reduce response conflict (Grison \& Strayer, 2001; Strayer \& Grison, 1999). However, the designs they used involved only repeated incompatible distractors. Hence, it was not possible to distinguish whether the repetition of stimuli (as targets) increased the level of activation of memory representations or whether it increased the connection with their respective responses. In this respect, rather than being in contradiction, our study supports and extends the ideas of Strayer and Grison on the role of response conflict in producing NP.

\section{Negative Priming: Selection Processes or Retrieval Processes?}

We explain our data in terms of neural mechanisms of selective attention, in particular the reactive inhibition of activated, but task-irrelevant or disruptive representations (Houghton \& Tipper, 1996; Strayer \& Grison, 1999). It was on the basis of such ideas about cognitive control, along with behavioral and psychophysiological data showing response activation from incompatible nontarget stimuli, that we first questioned the apparently widespread belief that response selection had been shown to play no sig- 
nificant role in NP. Furthermore, the specific predictions we tested in the experiments were derived from this same source. As far we know, our results are new and were not predicted by any other approach to NP.

Given this background, our discussion naturally hinges on events occurring during the processing of the prime display. As Tipper (2001) points out, behavioral priming measures require both a prime and a probe, and a full account needs to address both events, as well as the interaction between them. Other researchers consequently prefer to analyze NP in terms of hypothetical retrieval processes induced by the probe (Neill et al., 1992). The existence of this alternative approach has caused some debate (Milliken et al., 1998; Tipper, 2001), which we will not address here, since the retrieval-based accounts played no role in motivating our experiments. The only thing we manipulated was the selection problems facing participants in the prime displays. In concentrating on the probe, retrieval accounts do not specify what it means for a distractor stimulus to be ignored, how it is selected against, or how it may be "tagged" (Neill et al., 1992) in a way that is different from a target (Tipper, 2001). Obviously, experimental stimuli do not enter the brain labeled "target," "distractor," "do not respond," or anything else; rather it is only the interaction of their neural representations with the goal-based attentional state of the participant that establishes the behavioral relevance of a stimulus. Hence, we necessarily seek an explanation of these results in terms of selective attention - that is, the way in which the brain controls the state of activation and suppression of competing representations by reference to an internally represented goal.

\section{Conclusion}

NP due to an ignored, fleetingly presented, distractor object was first reported by Tipper (1985; previous reports of such an effect had been confined to Stroop words in which there is no separate object). In Tipper's study, participants were shown displays containing two superimposed line drawings - one in red, one in green - and had to respond to just one of them, identified by its color. The distractor stimuli were always associated with a response incompatible with that to the target. Nevertheless, the great majority of studies using the same or similar paradigms, and, with the recent exception of Strayer and Grison (1999), all accounts of the effect have concentrated on processes involving the representation of the stimulus. In this article, we have argued that previous studies purportedly showing NP without any involvement of response suppression have not demonstrated that it is not a factor in designs in which response competition is present (indeed, they could not logically have done so). In the present experiments, we have shown that NP can arise from more than one source, that these sources are dissociable, that it can be produced by response factors alone without stimulus repetition, and that what we have termed conventional NP may be a combination of more than one effect, the different sources combining in different proportions depending on the particular locus of the selection difficulties induced by the experimental context.

\section{REFERENCES}

Banich, M. T., Milham, M. P., Atchley, R., Cohen, N. J., Webb, A., WszaleK, T., ET AL. (2000). fMRI studies of Stroop tasks reveal unique roles of anterior and posterior brain systems in attentional selection. Journal of Cognitive Neuroscience, 12, 988-1000.

BAYLIS, G. C., \& BAYLIS, L. L. (2001). Visually misguided reaching in Balint's syndrome. Neuropsychologia, 39, 865-875.

Bowman, H., Schlaghecken, F., \& Eimer, M. (2006). A neural network model of inhibitory processes in subliminal priming. Visual Cognition, 13, 401-480.

Bruin, K. J., Kenemans, J. L., Verbaten, M. N., \& Van der Heijden, A. H. C. (2000). Habituation: An event-related potential and dipole source analysis study. International Journal of Psychophysiology, 36, 199-209.

Caputo, G., \& Guerra, S. (1998). Attentional selection by distractor suppression. Vision Research, 38, 669-689.

Cepeda, N. J., Cave, K. R., Bichot, N. P., \& Kim, M.-S. (1998). Spatial selection via feature-driven inhibition of distractor locations. Perception \& Psychophysics, 60, 727-746.

Claridge, G. S., Clark, K. H., \& Beech, A. R. (1992). Lateralization of the "negative priming" effect: Relationships with schizotypy and with gender. British Journal of Psychology, 83, 13-23.

Coles, M. G. H., Gratton, G., Bashore, T. R., Eriksen, C. W., \& Donchin, E. (1985). A psychophysiological investigation of the continuous flow model of human information processing. Journal of Experimental Psychology: Human Perception \& Performance, 11, 529-553.

Dalrymple-Alford, E. C., \& Budayr, B. (1966). Examination of some aspects of the Stroop color-word test. Perceptual \& Motor Skills, 23, 1211-1214.

De Jong, R., Liang, C.-C., \& Lauber, E. (1994). Conditional and unconditional automaticity: A dual-process model of effects of spatial stimulus-response correspondence. Journal of Experimental Psychology: Human Perception \& Performance, 20, 731-750.

Desimone, R., \& Duncan, J. (1995). Neural mechanisms of selective visual attention. Annual Review of Neuroscience, 18, 193-222.

DeSoto, M. C., Fabiani, M., Geary, D. C., \& Gratton, G. (2001). When in doubt, do it both ways: Brain evidence of the simultaneous activation of conflicting motor responses in a spatial Stroop task. Journal of Cognitive Neuroscience, 13, 523-536.

Duncan, J., \& Humphreys, G. W. (1989). Visual search and stimulus similarity. Psychological Review, 96, 433-458.

EIMER, M. (1999). Facilitatory and inhibitory effects of masked prime stimuli on motor activation and behavioural performance. Acta Psychologica, 101, 293-313.

Eimer, M., \& SCHLAGHeCKen, F. (1998). Effects of masked stimuli on motor activation: Behavioral and electrophysiological evidence. Journal of Experimental Psychology: Human Perception \& Performance, 24, 1737-1747.

Eriksen, B. A., \& EriKsen, C. W. (1974). Effect of noise letters upon the identification of a target letter in a nonsearch task. Perception \& Psychophysics, 16, 143-149.

Eriksen, C. W., Coles, M. G. H., Morris, L. R., \& O’Hara, W. P. (1985). An electromyographic examination of response competition. Bulletin of the Psychonomic Society, 23, 165-168.

Fox, E. (1994). Interference and negative priming from ignored distractors: The role of selection difficulty. Perception \& Psychophysics, 56, 565-574.

Fox, E. (1995). Negative priming from ignored distractors in visual selection: A review. Psychonomic Bulletin \& Review, 2, 145-173.

Gernsbacher, M. A., \& Faust, M. E. (1991). The mechanism of suppression: A component of general comprehension skill. Journal of Experimental Psychology: Learning, Memory, \& Cognition, 17, 245262.

Grison, S., \& StRAYER, D. L. (2001). Negative priming and perceptual fluency: More than what meets the eye. Perception \& Psychophysics, 63, 1063-1071. 
Hasher, L., Stoltzfus, E. R., Zacks, R. T., \& Rypma, B. (1991). Age and inhibition. Journal of Experimental Psychology: Learning, Memory, \& Cognition, 17, 163-169.

Hazeltine, E., Poldrack, R., \& Gabrieli, J. D. E. (2000). Neural activation during response competition. Journal of Cognitive Neuroscience, 12, 118-129.

Heil, M., Osman, A., Wiegelmann, J., Rolke, B., \& Hennighausen, E. (2000). N200 in the Eriksen-task: Inhibitory executive processes? Journal of Psychophysiology, 14, 218-225.

Houghton, G., \& Tipper, S. P. (1994). A model of inhibitory mechanisms in selective attention. In D. Dagenbach \& T. H. Carr (Eds.), Inhibitory processes in attention, memory, and language (pp. 53-112). San Diego: Academic Press.

Houghton, G., \& TiPPER, S. P. (1996). Inhibitory mechanisms of neural and cognitive control: Applications to selective attention and sequential action. Brain \& Cognition, 30, 20-43.

Houghton, G., \& Tipper, S. P. (1999). Attention and the control of action: An investigation of the effects of selection on population coding of hand and eye movement. In D. Heinke, G. W. Humphreys, \& A. Olson (Eds.), Connectionist models in cognitive neuroscience: Proceedings of the 5th Neural Computation and Psychology Workshop (pp. 283-298). London: Springer.

Houghton, G., Tipper, S. P., Weaver, B., \& Shore, D. I. (1996). Inhibition and interference in selective attention: Some tests of a neural network model. Visual Cognition, 3, 119-164.

Jonides, J., Smith, E. E., Marshuetz, C., Koeppe, R. A., \& ReuterLORENZ, P. A. (1998). Inhibition in verbal working memory revealed by brain activation. Proceedings of the National Academy of Sciences, 95, 8410-8413.

Kane, M. J., Hasher, L., Stoltzfus, E. R., Zacks, R. T., \& ConNELLY, S. L. (1994). Inhibitory attentional mechanisms and aging. Psychology \& Aging, 9, 103-112.

Leung, H.-C., Skudlarski, P., Gatenby, J. C., Peterson, B. S., \& GoRE, J. C. (2000). An event-related functional MRI study of the Stroop color word interference task. Cerebral Cortex, 10, 552-560.

Lowe, D. G. (1979). Strategies, context, and the mechanism of response inhibition. Memory \& Cognition, 7, 382-389.

Lowe, D. G. (1985). Further investigations of inhibitory mechanisms in attention. Memory \& Cognition, 13, 74-80.

MaCLEOD, C. M. (1991). Half a century of research on the Stroop effect: An integrated review. Psychological Bulletin, 109, 163-203.

Malley, G. B., \& Strayer, D. L. (1995). Effect of stimulus repetition on positive and negative identity priming. Perception \& Psychophysics, 57, 657-667.

MARCEL, A. J. (1983). Conscious and unconscious perception: Experiments on visual masking and word recognition. Cognitive Psychology, 15, 197-237.

Marí-Beffa, P., Estévez, A. F., \& Danziger, S. (2000). Stroop interference and negative priming: Problems with inferences from null results. Psychonomic Bulletin \& Review, 7, 499-503.

Marí-Beffa, P., Fuentes, L. J., Catena, A., \& Houghton, G. (2000). Semantic priming in the prime task effect: Evidence of automatic semantic processing of distractors. Memory \& Cognition, 28, 635-647.

Marí-Beffa, P., Houghton, G., Estévez, A. F., \& Fuentes, L. J. (2000). Word-based grouping affects the prime task effect on semantic priming. Journal of Experimental Psychology: Human Perception \& Performance, 26, 469-479.

Metzler, C., \& Parkin, A. J. (2000). Reversed negative priming following frontal lobe lesions. Neuropsychologia, 38, 363-379.

MilLER, J. (1991). The flanker compatibility effect as a function of visual angle, attentional focus, visual transients, and perceptual load: A search for boundary conditions. Perception \& Psychophysics, 49, 270-288.

Milliken, B., Joordens, S., Merikle, P. M., \& Seiffert, A. E. (1998). Selective attention: A reevaluation of the implications of negative priming. Psychological Review, 105, 203-229.

NEILL, W. T. (1977). Inhibitory and facilitatory processes in attention. Journal of Experimental Psychology: Human Perception \& Performance, 3, 444-450.

Neill, W. T., Terry, K. M., \& Valdes, L. A. (1994). Negative prim- ing without probe selection. Psychonomic Bulletin \& Review, 1, 119121

Neill, W. T., Valdes, L. A., Terry, K. M., \& Gorfein, D. S. (1992). Persistence of negative priming: II. Evidence for episodic trace retrieval. Journal of Experimental Psychology: Learning, Memory, \& Cognition, 18, 993-1000.

Neill, W. T., \& WestberRy, R. L. (1987). Selective attention and the suppression of cognitive noise. Journal of Experimental Psychology: Learning, Memory, \& Cognition, 13, 327-334.

Neumann, E., \& DeSchepper, B. G. (1992). An inhibition-based fan effect: Evidence for an active suppression mechanism in selective attention. Canadian Journal of Psychology, 46, 1-40.

Nievas, F., \& Marí-Beffa, P. (2002). Negative priming from the nonselected meaning of the homograph. British Journal of Psychology, 93, 47-66.

Orekhova, E. V., Stroganova, T. A., \& Posikera, I. N. (2001). Alpha activity as an index of cortical inhibition during sustained internally controlled attention in infants. Clinical Neurophysiology, 112, 740749

PliszKa, S. R., Liotti, M., \& WoldorfF, M. G. (2000). Inhibitory control in children with attention-deficit/hyperactivity disorder: Eventrelated potentials identify the processing component and timing of an impaired right-frontal response-inhibition mechanism. Biological Psychiatry, 48, 238-246.

Praamstra, P., \& Plat, F. M. (2001). Failed suppression of direct visuomotor activation in Parkinson's disease. Journal of Cognitive Neuroscience, 13, 31-43.

Read, L. E., \& Proctor, R. W. (2004). Spatial stimulus-response compatibility and negative priming. Psychonomic Bulletin \& Review, 11, 41-48.

Shiu, L.-P., \& Kornblum, S. (1996). Negative priming and stimulusresponse compatibility. Psychonomic Bulletin \& Review, 3, 510-514.

StraYer, D. L., \& Grison, S. (1999). Negative identity priming is contingent on stimulus repetition. Journal of Experimental Psychology: Human Perception \& Performance, 25, 24-38.

TIPPER, S. P. (1985). The negative priming effect: Inhibitory effects of ignored primes. Quarterly Journal of Experimental Psychology, 37A 571-590.

TIPPER, S. P. (1991). Less attentional selectivity as a result of declining inhibition in older adults. Bulletin of the Psychonomic Society, 29, 45-47.

TIPPER, S. P. (2001). Does negative priming reflect inhibitory mechanisms? A review and integration of conflicting views. Quarterly Journal of Experimental Psychology, 54A, 321-343.

Tipper, S. P., Brehaut, J. C., \& Driver, J. (1990). Selection of moving and static objects for the control of spatially directed action. Journal of Experimental Psychology: Human Perception \& Performance, 16, 492-504.

Tipper, S. P., \& Cranston, M. (1985). Selective attention and priming: Inhibitory and facilitatory effects of ignored primes. Quarterly Journal of Experimental Psychology, 37A, 591-611.

TipPer, S. P., Eissenberg, T., \& WeAVER, B. (1992). The effects of practice on mechanisms of attention. Bulletin of the Psychonomic Society, 30, $77-80$

Tipper, S. P., Howard, L., \& Houghton, G. (2000). Behavioral consequences of selection from neural population codes. In S. Monsell \& J. Driver (Eds.), Control of cognitive processes: Attention and performance XVIII (pp. 223-246). Cambridge, MA: MIT Press.

TIPPER, S. P., HowARD, L., \& JACKSON, S. R. (1997). Selective reaching to grasp: Evidence for distractor interference effects. Visual Cognition, 4, $1-38$

Tipper, S. P., MacQueen, G. M., \& Brehaut, J. C. (1988). Negative priming between response modalities: Evidence for the central locus of inhibition in selective attention. Perception \& Psychophysics, 43, 45-52.

Tipper, S. P., Weaver, B., Cameron, S., Brehaut, J. C., \& Bastedo, J. (1991). Inhibitory mechanisms of attention in identification and localization tasks: Time course and disruption. Journal of Experimental Psychology: Learning, Memory, \& Cognition, 17, 681-692.

Tipper, S. P., Weaver, B., \& Houghton, G. (1994). Behavioral goals 
determine inhibitory mechanisms of selective attention. Quarterly Journal of Experimental Psychology, 47A, 809-840.

Tipper, S. P., Weaver, B., KirkPatrick, J., \& Lewis, S. (1991). Inhibitory mechanisms of attention: Locus, stability, and relationship with distractor interference effects. British Journal of Psychology, 82, 507-520.

UNDERWOOD, G. (1976). Semantic interference from unattended printed words. British Journal of Psychology, 67, 327-338.

Watson, D. G., \& Humphreys, G. W. (1997). Visual marking: Prioritiz- ing selection for new objects by top-down attentional inhibition of old objects. Psychological Review, 104, 90-122.

West, R., \& Alain, C. (2000). Age-related decline in inhibitory control contributes to the increased Stroop effect in older adults. Psychophysiology, 37, 179-189.

(Manuscript received June 18, 2004;

revision accepted for publication February 21, 2005.) 